PSU/TH/187

July 1997

\title{
Third-generation leptoquark decays and collider searches
}

\author{
M. A. Doncheski \\ Department of Physics \\ The Pennsylvania State University \\ Mont Alto, PA 17237 USA \\ and \\ R. W. Robinett \\ Department of Physics \\ The Pennsylvania State University \\ University Park, PA 16802 USA
}

\begin{abstract}
Collider searches for first-, second-, and third-generation scalar $(S)$ or vector $(V)$ leptoquarks (LQs) focus on the quark-lepton decay modes $S, V \rightarrow q l$. For $S U(2)$-doublet and -triplet leptoquarks with a sufficiently large splitting between the components, decays involving real $W$-boson emission (such as $S_{2}^{(+5 / 3)} \rightarrow$ $S_{2}^{(+2 / 3)} W^{+}$and others) become possible and can change the patterns of leptoquark decays. For third-generation leptoquarks, where these mass splittings might be large, such modes could dominate certain leptoquark decays as they are (if kinematically allowed) guaranteed to be of order $g^{2}$ where $g$ is the electroweak coupling. We calculate the decay rates for all such processes involving $S U(2)$-doublet and triplet, scalar and vector leptoquarks. Standard limits on mass splittings from precision electroweak measurements imply that only such decays involving $S U(2)$-doublet scalar LQs are likely kinematically possible.
\end{abstract}


The recent observation by two groups at HERA [1] of an excess of events in deep inelastic scattering at large $Q^{2}$ has generated a large number of possible explanations in terms of new particles beyond the standard model. One of the most widely discussed of these scenarios has been the possibility that relatively low mass leptoquarks (LQs) are responsible and by now many aspects of this idea have been studied in some detail. (For recent reviews of some of the main ideas and references to many of the original papers, see Refs. [2]-[6].)

All such proposals have to deal with limits on leptoquark masses arising from LQ pair-production processes at colliders. These limits rely solely on the LQ electroweak and/or strong couplings (which are restricted in form) and not (directly) on the unknown LQ-quark-lepton couplings; the only assumption used is that the pair-produced LQs decay via $L Q \rightarrow q l$. Limits from $e^{+} e^{-}$collisions below [7] and at the $Z^{0}$ [8] have excluded leptoquarks with masses below $\sim 45 \mathrm{GeV}$, while searches in $p \bar{p}$ collisions for first- [9], second- [10], and third-generation [11] leptoquarks have set increasingly stringent lower bounds on LQ masses. For example, recent analyses of TEVATRON data have yielded limits on first-generation scalar leptoquarks of $M\left(L Q_{1}\right)>210 \mathrm{GeV}$ [12] and $M\left(L Q_{1}\right)>240 \mathrm{GeV}$ [13] (assuming a branching ratio to $e q$ final states of $\beta=1.0)$ and $M\left(L Q_{1}\right)>147 \mathrm{GeV}(71 \mathrm{GeV})$ [14 for $\beta=0.5(0.0)$ where the $\beta=0.0$ limit is important as it provides a constraint on LQs which couple exclusively to $\nu q$ final states. Such analyses by now routinely include the effect of NLO corrections to LQ pair-production processes [15] and are also usually extended to provide limits on vector leptoquarks (which have a larger strong-interaction cross-section at leading order); for example, one finds that $M\left(L Q_{1}\right)>298 \mathrm{GeV}(270 \mathrm{GeV})$ for $\beta=1.0(0.5)$ [14] for first-generation vector LQs using the same assumptions about decay modes. 
Second- and third-generation LQ searches also rely on the $q l$ decay signal with $\mu$-jet and $\tau$ - jet (with one jet identified as arising from a $b$-quark) final states studied respectively. Such signals are also the ones touted for future extensions of these methods to LHC energies [16].

The authors of Ref. [6] have pointed out that if the splitting between the components of an $S U(2)$-doublet scalar LQ is large enough, then processes involving real $W$-boson emission become kinematically possible and can easily dominate the decay rates for such leptoquarks. Since this possibility is not restricted (in principle) to either $S U(2)$ doublets or scalar LQs, in this note we generalize and elaborate on this comment by calculating the decay rates involving real $W$-boson emission for all $S U(2)$-doublet and -triplet leptoquarks, for both the scalar and vector cases. Since this is arguably most likely for the third-generation (if the pattern of quark doublets is any guide) we will focus on that case explicitly. In this case, the main decay mode of some of the leptoquarks we consider would be $S \rightarrow t l$ involving the heavy top as the final state quark and this decay would also be somewhat suppressed by phase space factors, further enhancing any new decay modes.

To determine to what extent such mass splittings are allowed, we also examine the standard analyses of the contributions of such splittings to such precision electroweak observables as the $\rho$ parameter. (Several analyses involving other precision electroweak parameters have already appeared [5], [17] and give similar bounds to those we discuss here.) One must always bear in mind that models which contain one or the other type of leptoquarks which we consider here may well also predict many other new particles which would contribute to such constraints and make such mass splittings less likely. We will consider the limits on $\Delta \rho$ which arise from the addition of only 
one variety of leptoquark beyond the standard model at a time and we find that mass splittings sufficient to allow for such decays (namely $m_{2} \geq m_{1}+M_{W}$ ) are likely only possible for scalar doublets. Babu, Kolda, and March-Russell [6] note, however, that mixings between various leptoquarks can significantly weaken such constraints so, for completeness, we present the complete set of decay rates for all cases which are considered in the standard $S U(3) \times S U(2) \times U(1)$ analyses 18 of leptoquark quantum numbers. Specifically, we will consider the $S U(2)$-doublet and -triplet leptoquarks, which in the notation of Ref. [4] have charges

\begin{tabular}{cl} 
leptoquark & \multicolumn{1}{c}{$Q$} \\
\hline$S_{2}$ & $(5 / 3,2 / 3)$ \\
$\tilde{S}_{2}$ & $(2 / 3,-1 / 3)$ \\
$S_{3}$ & $(4 / 3,1 / 3,-2 / 3)$ \\
\hline$V_{2}$ & $(4 / 3,1 / 3)$ \\
$\tilde{V}_{2}$ & $(1 / 3,-2 / 3)$ \\
$V_{3}$ & $(5 / 3,2 / 3,-1 / 3)$ \\
\hline
\end{tabular}

The couplings to third-generation quarks and leptons is cataloged in Ref. [5].

We first consider the $W$-boson decays involving $S U(2)$ doublet, scalar leptoquarks, such as

$$
S \rightarrow s W^{+}
$$

which would compete, for example, with the ordinary $q l$ decays

$$
S \rightarrow \tau^{+} q
$$

where $(S, s, q)$ can be $\left(S_{2}^{(+5 / 3)}, S_{2}^{(+2 / 3)}, t\right)$ or $\left(\tilde{S}^{(+2 / 3)}, \tilde{S}_{2}^{(-1 / 3)}, b\right)$. In order to first determine to what extent a mass splitting large enough to permit such decays is still allowed, we recall that the contribution of a single $S U(2)$-doublet scalar leptoquark to the $\rho$ parameter is

$$
\Delta \rho=\frac{1}{2}\left[\frac{3 G_{F}}{8 \sqrt{2} \pi^{2}}(\Delta m)^{2}\right]
$$


where

$$
(\Delta m)^{2}=m_{1}^{2}+m_{2}^{2}-\frac{m_{1}^{2} m_{2}^{2}}{m_{1}^{2}-m_{2}^{2}} \log \left(\frac{m_{1}^{2}}{m_{2}^{2}}\right)
$$

This contribution is similar to that from scalar quarks in supersymmetry [19] (either one set of left or right-handed squarks) and equal to half that of a standard chiral fermion $S U(2)$-doublet [20]. A fit to precision electroweak data from the most recent Particle Data Group compilation [21] gives the bounds [22]

$$
(\Delta m)^{2} \leq(108 \mathrm{GeV})^{2},(139 \mathrm{GeV})^{2},(172 \mathrm{GeV})^{2}
$$

for $M_{H}=60 \mathrm{GeV}, 300 \mathrm{GeV}$, and $1000 \mathrm{GeV}$ respectively where $M_{H}$ is the mass of the standard model Higgs boson. For small mass splittings satisfying $m_{2}-m_{1}<<m_{2}+m_{1}$, we approximate $(\Delta m)^{2} \approx 4\left(m_{2}-m_{1}\right)^{2} / 3$ so that we have

$$
m_{2}-m_{1} \lesssim 95 \mathrm{GeV}, 120 \mathrm{GeV}, 149 \mathrm{GeV}
$$

Thus, in what follows, for $S U(2)$-doublet scalar leptoquarks we will assume that

$$
M(S)-M(s) \equiv M-m=100 \mathrm{GeV}, 125 \mathrm{GeV}, 150 \mathrm{GeV}
$$

are all allowed.

The decay rate for either of the $W$-emission processes in Eqn. (2) can be written in the form

$$
\Gamma\left(S \rightarrow s W^{+}\right)=\frac{g^{2}}{32 \pi}\left(\frac{M^{3}}{M_{W}^{2}}\right) \lambda^{3 / 2}\left(1, m^{2} / M^{2}, M_{W}^{2} / M^{2}\right)
$$

where the familiar kinematic function $\lambda(x, y, z)$ is given by

$$
\lambda(x, y, z)=x^{2}+y^{2}+z^{2}-2 x y-2 x z-2 y z
$$


and $M, m$ are the masses of the heavier, lighter leptoquark respectively. For comparison, the standard LQ decay is

$$
\Gamma\left(S \rightarrow q \tau^{+}\right)=\frac{g_{R, L}^{2}}{16 \pi} M\left(1-\frac{M_{q}^{2}}{M^{2}}\right)^{2}
$$

with $M_{q}=M_{t}$ for the heavy top and $M_{q} \approx 0$ for the decays involving $\tau b$ final states. The ratio of these two decay rates can be written in the form

$$
R=\frac{\Gamma\left(S \rightarrow s W^{+}\right)}{\Gamma\left(S \rightarrow q \tau^{+}\right)}=\frac{1}{2}\left(\frac{g}{g_{R, L}}\right)^{2}\left(\frac{M^{2}}{M_{W}^{2}}\right) \frac{\lambda^{3 / 2}\left(1, m^{2} / M^{2}, M_{W}^{2} / M^{2}\right)}{\left(1-M_{q}^{2} / M^{2}\right)^{2}}
$$

We plot this ratio in Fig. 1 without the overall factor of $\left(g / g_{R, L}\right)^{2}$ for both the case where the final-state quark is heavy $(q=t)$ or light $(q=b)$ to show the effect of the top-quark threshold: we show three cases consistent with the mass splittings in Eqn. (8). We note that even without any large ratio of coupling constants, the $W$-boson emission decay process can easily be competitive or even dominate the standard $q l$ decay process. For first-generation HERA-inspired leptoquarks where the LQ couplings are of order $g_{L, R} \approx 0.002-0.0005$ (depending on whether it is produced form a valence $u$ - or $d$ type quark [4]), the ratio of couplings can be as large as $\left(g / g_{R, L}\right)^{2} \approx 200-800$. For third-generation LQs, however, the couplings are not constrained by rare processes [23] and so could be larger and therefore the ratio of couplings much smaller; however, even with an LQ coupling of electromagnetic strength, namely $g_{R, L}=e$, this ratio of couplings still provides a factor of $(g / e)^{2}=1 / \sin ^{2}\left(\theta_{W}\right) \approx 4$ enhancement.

For $S U(2)$-triplet scalar leptoquarks, the decay rate in Eqn. (9) is multiplied by a factor of 2 , but the constraints from precision electroweak measurements are now also more stringent. For example, the contribution to $\Delta \rho$ is increased so that the constraint in Eqn. (6) now becomes

$$
2\left[\left(\Delta m_{(+, 0)}\right)^{2}+\left(\Delta m_{(0,-)}\right)^{2}\right] \leq(108 G e V)^{2},(139 G e V)^{2},(172 G e V)^{2}
$$


where the $+, 0,-$ subscripts refer to the three components of the iso-triplet. If we assume, for simplicity, that $m_{+}-m_{0}=m_{0}-m_{-}=\delta m$ and that $\delta m<<m_{+}, m_{0}, m_{-}$, we find that this limit translates into the constraint

$$
\delta m \lesssim \sqrt{\frac{3}{16}}(108 \mathrm{GeV}, 139 \mathrm{GeV}, 172 \mathrm{GeV}) \approx(47 \mathrm{GeV}, 60 \mathrm{GeV}, 75 \mathrm{GeV})
$$

so that the $W$-emission process is not kinematically allowed.

We next turn to decays of vector leptoquarks involving $W$-emission, considering first such $S U(2)$-doublet processes as

$$
V \rightarrow v W^{+}
$$

which will be compared to the more standard $q l$ decays such as

$$
V \rightarrow \tau^{+} \bar{q}
$$

where $(V, v, q)$ can be $\left(V_{2}^{(+4 / 3)}, V_{2}^{(+1 / 3)}, b\right)$ or $\left(\tilde{V}_{2}^{(+1 / 3)}, \tilde{V}_{2}^{(-2 / 3)}, t\right)$. This case is, in principle, interesting since the decay of heavy vector particles into pairs of vectors can exhibit extra enhancements given by ratios of masses; examples include the decays of heavy gauge bosons into weak bosons such as $Z^{\prime} \rightarrow W^{+} W^{-}$[24] or $Z^{\prime} \rightarrow W^{+} W^{-} Z^{0}$ [25] in extended gauge theories or string-inspired models. We find that this is indeed the case as the decay rate for vector leptoquarks via $W$-emission in either of the cases in Eqn. (15) is

$$
\begin{aligned}
\Gamma\left(V \rightarrow v W^{+}\right)= & \frac{g^{2}}{384 \pi}\left(\frac{M^{5}}{m^{2} M_{W}^{2}}\right) \lambda^{1 / 2}\left(1, m^{2} / M^{2}, M_{W}^{2} / M^{2}\right) \\
& \times\left(1-\frac{\left(m-M_{W}\right)^{2}}{M^{2}}\right)\left(1-\frac{\left(m+M_{W}\right)^{2}}{M^{2}}\right) \\
& \times\left[1+\frac{m^{4}}{M^{4}}+\frac{M_{W}^{4}}{M^{4}}+10 \frac{m^{2}}{M^{2}}+10 \frac{M_{W}^{2}}{M^{2}}+10 \frac{m^{2} M_{W}^{2}}{M^{4}}\right]
\end{aligned}
$$


In the limit that $m=M_{W}$, the decay kinematics reduces to that of the $Z^{\prime} \rightarrow W^{+} W^{-}$ case mentioned above, enhanced by mass ratios of the form $\left(M^{2} / m M_{W}\right)^{2}$, but without the decrease in rate due to $Z^{\prime} \longleftrightarrow Z^{0}$ mixing angles. The standard $q l$ decay is

$$
\Gamma\left(V \rightarrow \bar{q} \tau^{+}\right)=\frac{g_{R, L}^{2}}{24 \pi} M\left(1-\frac{M_{q}^{2}}{M^{2}}\right)^{2}\left(1+\frac{M_{q}^{2}}{2 M^{2}}\right)
$$

where $l$ is a massless lepton.

In this case, however, the constraints from contributions to the $\rho$ parameter are more stringent; for each $S U(2)$-doublet vector leptoquark the right-hand-side of Eqn. (6) is replaced by $3(\Delta m)^{2}$ so that the mass-splittings must satisfy

$$
m_{2}-m_{1} \lesssim \frac{1}{2}(108 \mathrm{GeV}, 139 \mathrm{GeV}, 172 \mathrm{GeV}) \approx(54 \mathrm{GeV}, 70 \mathrm{GeV}, 86 \mathrm{GeV})
$$

so that $W$-emission decays are allowed only for a very limited region of the allowed parameter space.

Once again, we plot the ratio of the two decay rates in Eqns. (17) and (18) in Fig. 2 without the overall factor of $\left(g / g_{R, L}\right)^{2}$, this time using the values $M-m=85 \mathrm{GeV}$ (which is allowed under our assumptions) and $M-m=100 \mathrm{GeV}$ (which is not, but does show the enhanced decay rates possible in this mode by comparison with Fig. 1.) If the bounds on mass splittings due to $\rho$ parameter constraints were indeed weakened by mixing phenomena as in Ref. [6], this decay could be dramatically enhanced.

Finally, for the case of $S U(2)$-triplet, vector LQ decays, such as $V_{3}^{(+5 / 3)} \rightarrow V_{3}^{(+2 / 3)} W^{+}$ or $V_{3}^{(+2 / 3)} \rightarrow V_{3}^{(-1 / 3)} W^{+}$, the decay rates in Eqn. (17) are increased by a factor of 2 , but the $\rho$ parameter constraints are even more restrictive as one requires that

$$
6\left[\left(\Delta m_{+, 0}\right)^{2}+\left(\Delta m_{0,-}\right)^{2}\right] \leq(108 G e V)^{2},(139 G e V)^{2},(172 G e V)^{2}
$$


or

$$
\delta m \lesssim 27 \mathrm{GeV}, 35 \mathrm{GeV}, 43 \mathrm{GeV}
$$

and presumably only mixing phenomena would allow for such constraints to relaxed.

In conclusion, we have examined the $W$-boson decay rates for all possible $S U(2)$ doublet and -triplet leptoquarks for both the scalar and vector cases. Using standard limits from precision electroweak measurements, if we ignore any possible mixing phenomena, only the $S U(2)$-doublet leptoquark scalar case would have this decay mode kinematically accessible. Interestingly, in that case, the main competing $q l$ decay mode involves the heavy top quark which suppresses the standardly assumed quark-lepton decay process. Finally, we note that the authors of Ref. [6] have also considered other electroweak decays (involving $Z^{0}$-boson emission) which are possible only if there is mixing present.

\section{Acknowledgments}

We thank T. Rizzo for communications about precision electroweak limits and leptoquarks and J. March-Russell for communications regarding Ref. [6]. One of us (M.A.D) acknowledges the support of Penn State University through a Research Development Grant (RDG). 


\section{References}

[1] C. Adloff et al. (H1 Collaboration), Z. Phys. C74, 191 (1997); J. Breitweg et al., Z. Phys. C74 (1997) 207.

[2] J. Blümlein, DESY-97-105, hep-ph/9706362.

[3] P. Frampton, hep-ph/9706220, to appear in the Proceedings of Beyond the Standard Model V, Balestrand, Norway, May 1997.

[4] Z. Kunszt and W. J. Stirling, DTP/97/16, hep-ph/9703427.

[5] J. L. Hewett and T. G. Rizzo, SLAC-PUB-7430, hep-ph/9703337.

[6] K. S. Babu, C. Kolda, and J. March-Russell, Institute for Advanced Study preprint IASSNS-HEP-97-64, hep-ph/9705414.

[7] W. Bartelt et al. (JADE Collaboration), Z. Phys. C36 (1987) 15 ; G. N. Kim et al. (AMY Collaboration), Phys. Lett. B240 (1990) 243.

[8] B. Adeva et al. (L3 Collaboration), Phys. Lett. B261 (1991) 169; G. Alexander et al. (OPAL Collaboration), Phys. Lett. B263 (1991) 123; P. Abreu et al. (DELPHI Collaboration), Phys. Lett. B275 (1992) 222; D. Decamp et al. (ALEPH Collaboration), Phys. Rep. 216 (1992) 253.

[9] J. Alitti et al. (UA2 Collaboration), Phys. Lett, B274 (1992) 507; F. Abe et al. (CDF Collaboration), Phys. Rev. D48 (1993) R3939; S. Abachi et al. (D0 Collaboration), Phys. Rev. Lett. 72, (1994) 965.

[10] F. Abe et al. (CDF Collaboration), Phys. Rev. Lett. 75 (1995) 1012; S. Abachi et al. (D0 Collaboration), Phys. Rev. Lett. 75 (1995) 3618. 
[11] F. Abe et al. (CDF Collaboration), Phys. Rev. Lett. 78 (1997) 2906.

[12] H. S. Kambara (for the CDF Collaboration), hep-ex/9706026, to appear in the Proceedings of the XII Hadron Collider Physics Symposium, June 1997, Stony Brook).

[13] M. Krämer, RAL-TR-97-033, hep-ph/9707422

[14] J. A. Valls (for the CDF and D0 Collaborations), FERMILAB-CONF-97-135-E, (to appear in the Proceedings of the XXXII Rencontres de Moriond, Les Arc, France, 1997).

[15] M. Krämer, T. Plehn, M. Spira, P.M. Zerwas. Phys. Rev. Lett. 79 (1997) 341.

[16] B. Dion, L. Marleau, G. Simon, and M. de Montigny, LAVAL-PH-96-17, hep-ph/9701285.

[17] E. Keith and E. Ma, UCRHEP-T188, hep-ph/9707214.

[18] W. Buchmüller, R. Rückl, and D. Wyler, Phys. Lett. B191 (1987) 442.

[19] R. Barbieri, M. Frigeni, F. Giuliani, and H. E. Haber, Nucl. Phys. B341 (1990) 309.

[20] M. S. Chanowitz, M. A. Furman, and I. Hinchliffe, Nucl. Phys. B153 (1979) 402.

[21] R. M. Barnett et al., Review of Particle Properties, Phys. Rev. D54 (1996) 1.

[22] See Ref. [21], pp. 103-105.

[23] S. Davidson, D. Bailey, and B. A. Campbell, Z. Phys. C61 (1994) 613. 
[24] R. Robinett and J. L. Rosner, Phys. Rev. D25 (1982) 3036; (E) ibid. D27 (1983) 679.

[25] T. G. Rizzo and R. Robinett, Phys. Lett B226 (1989) 117. 


\section{Figure Captions}

Fig. 1. The ratio of the decay width $\Gamma\left(S \rightarrow s W^{+}\right)$to $\Gamma\left(S \rightarrow t \tau^{+}\right)$versus $M(S)$ (in $G e V)$ where $(S, s)$ are $S U(2)$-doublet leptoquark pairs such as $\left(S_{2}^{(+5 / 3)}, S_{2}^{(+2 / 3)}\right)$ or $\left(\tilde{S}_{2}^{(+2 / 3)}, \tilde{S}_{2}^{(-1 / 3)}\right)$. The dash (dot-dash, solid) curves correspond to a doublet splitting allowed by precision electroweak measurements corresponding to $M(S)-M(s)=150 \mathrm{GeV}(125 \mathrm{GeV}, 100 \mathrm{GeV})$. The upper curve in each case corresponds to assuming a decay involving the top quark, in which case we assume that $M_{t}=175 \mathrm{GeV}$; the lower curves assume a massless quark, for comparison, to better judge the effects of the top-quark production threshold.

Fig. 2. Same as Fig. 1, but for the vector leptoquark decays $\Gamma\left(V \rightarrow v W^{+}\right)$and $\Gamma(V \rightarrow$ $q l)$ where $l$ is a massless lepton. In this case we only consider a mass splitting $M(V)-M(v)=85 \mathrm{GeV}$ as allowed by precision electroweak data, but also show the case corresponding to $100 \mathrm{GeV}$ for comparison to the same case in Fig. 1 to show the enhancements possible in this vector decay mode. 


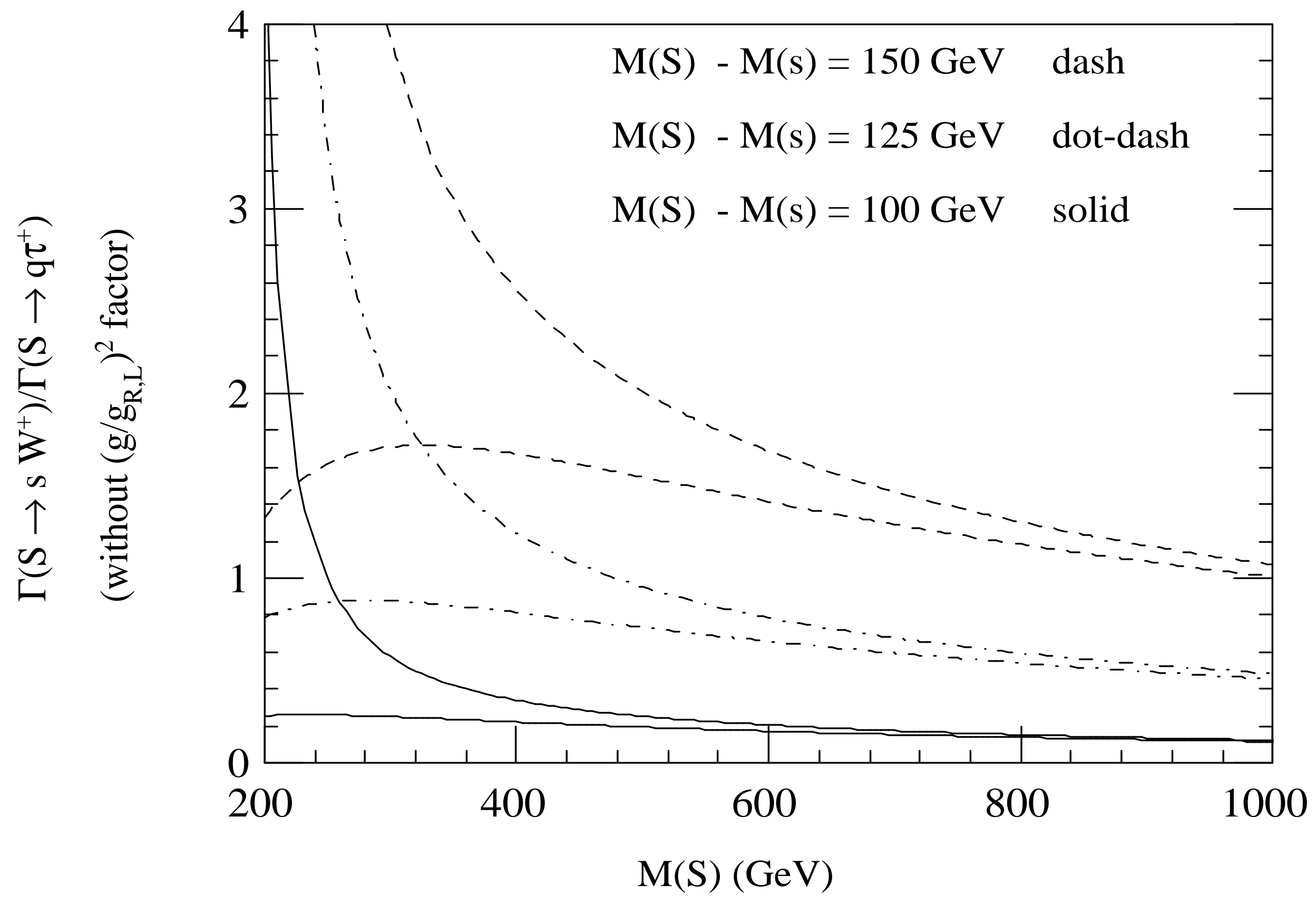




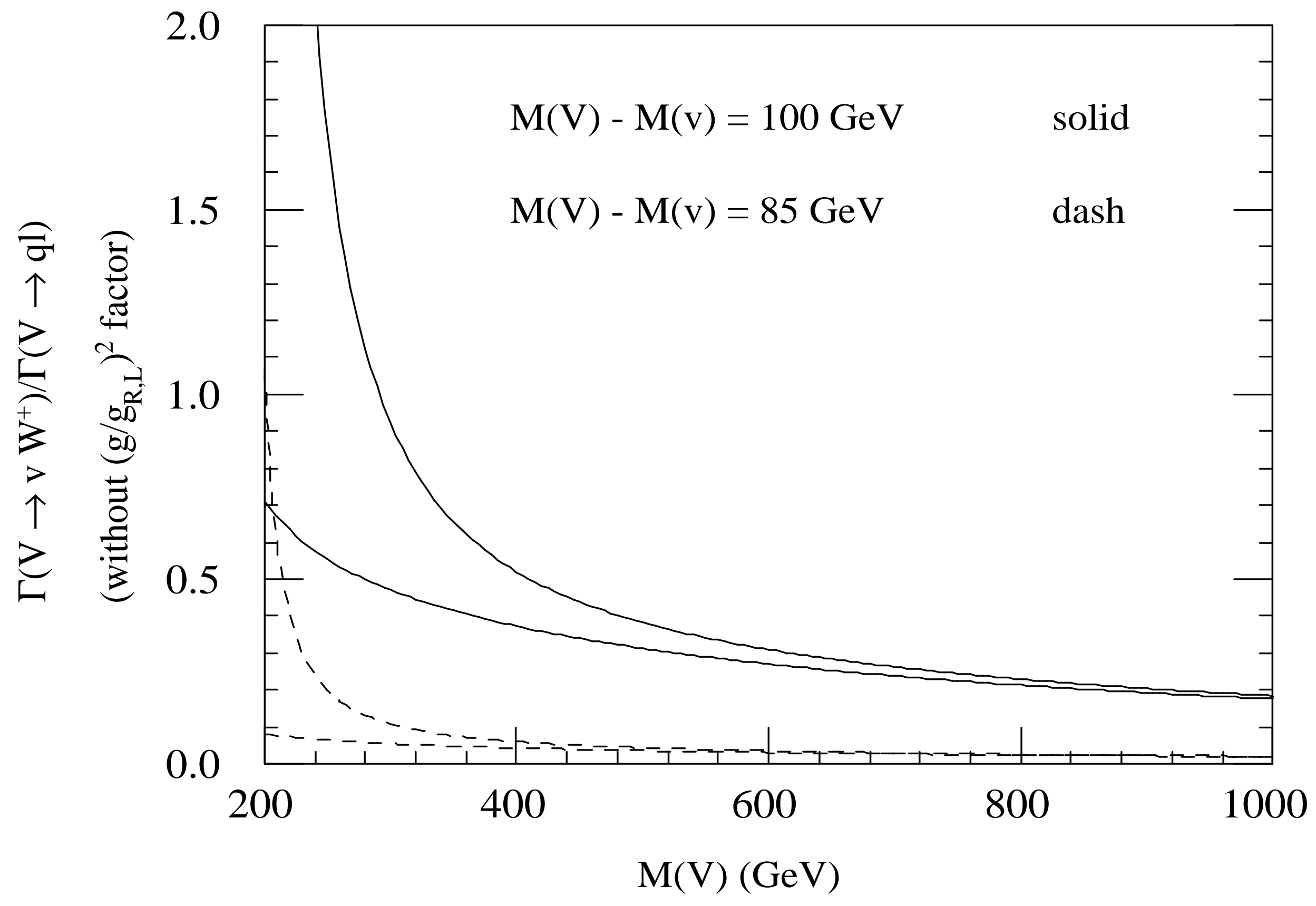

